\title{
Metabolic alterations and distribution of five-carbon precursors in jasmonic acid-elicited Catharanthus roseus cell suspension cultures
}

\author{
Mohd Zuwairi Saiman ${ }^{1,2} \cdot$ Natali Rianika Mustafa ${ }^{1,3} \cdot$ Young Hae Choi $^{1} \cdot$ \\ Robert Verpoorte $^{1} \cdot$ Anna Elisabeth Schulte ${ }^{1,3}$
}

Received: 25 November 2014/Accepted: 7 April 2015/Published online: 18 April 2015

(C) The Author(s) 2015. This article is published with open access at Springerlink.com

\begin{abstract}
In Catharanthus roseus cell cultures, the terpenoid moiety is considered as the limiting factor in terpenoid indole alkaloid (TIA) production. The pathway of terpenoidal precursors in TIA is strongly linked with other terpenoid pathways, suggesting that TIA production might be limited by competition for the five-carbon (C5) precursors. This raises the question whether the stimulation of TIA production by certain signal molecules is due to a redistribution of $\mathrm{C} 5$ precursors between the associated terpenoid pathways and/or to a total increase of the precursor availability. To investigate the effect of a TIA-increasing signal molecule on C5 distribution, the cell suspension cultures of $C$. roseus were elicited with jasmonic acid (JA) and the metabolic changes of different terpenoid pathways were evaluated targeting on TIA (monoterpenoid; C10), carotenoids (tetraterpenoid; C40), and phytosterols (triterpenoid; C30). The chromatographic analyses showed that TIA and carotenoid levels almost doubled upon JA elicitation, while phytosterol levels remained constant if compared to the controls. Apparently, both TIA and carotenoid routes benefit from an increased C5 flow in the methyl-erythritol phosphate pathway, and
\end{abstract}

Electronic supplementary material The online version of this article (doi:10.1007/s11240-015-0773-8) contains supplementary material, which is available to authorized users.

Natali Rianika Mustafa

mustafa@chem.leidenuniv.nl

1 Natural Products Laboratory, Institute of Biology, Leiden University, 2300 RA Leiden, The Netherlands

2 Institute of Biological Sciences, Faculty of Science, University of Malaya, 50603 Kuala Lumpur, Malaysia

3 ExPlant Technologies B.V., Galileiweg 8, 2333 BD Leiden, The Netherlands potential export of $\mathrm{C} 5$ precursors to the cytosolic terpenoid routes, e.g., towards the phytosterols is minimal. However, the relative composition of individual compounds within each group remains similar when comparing elicited and control cells. This suggests that the increased production of TIA and carotenoid upon JA elicitation is predominantly caused by an increase of the precursor availability rather than due to a redistribution of C5 precursors between the associated terpenoid pathways. Furthermore, NMR-based metabolomics analysis showed a discrimination of JAelicited and control cells between 24 and $72 \mathrm{~h}$ after treatments with significant changes in levels of strictosidine, malic acid, and sucrose. This study portrays metabolic alterations upon JA elicitation and channeling of C5 precursors in different terpenoid biosynthetic pathways, which provides a knowledge platform for developing strategies to engineer fluxes in a complex biosynthetic network in order to obtain high TIA-producing $C$. roseus cell lines.

Keywords Madagascar periwinkle $\cdot$ Jasmonate elicitation - Metabolic engineering - Terpenoid indole alkaloids · Carotenoids - Sterols - Biosynthetic pathway · NMR-based metabolomics

\section{Introduction}

Catharanthus roseus plants produce a diverse array of secondary metabolites including terpenoid indole alkaloids (TIAs), which are a valuable and unique source of the pharmaceutically important anticancer compounds vincristine and vinblastine. The other important TIAs produced by $C$. roseus are ajmalicine and serpentine having antihypertensive activity (van der Heijden et al. 2004). 
Fig. 1 Terpenoid biosynthetic pathway leading to different terpenoid groups and interaction between the precursor pools in cytosolic mevalonate pathway (MVA) and plastidial MEP pathway. IPP isopentenyl diphosphate, DMAPP dimethylallyl diphosphate, GPP geranyl diphosphate, $F P P$ farnesyl diphosphate, $G G P P$ geranylgeranyl diphosphate

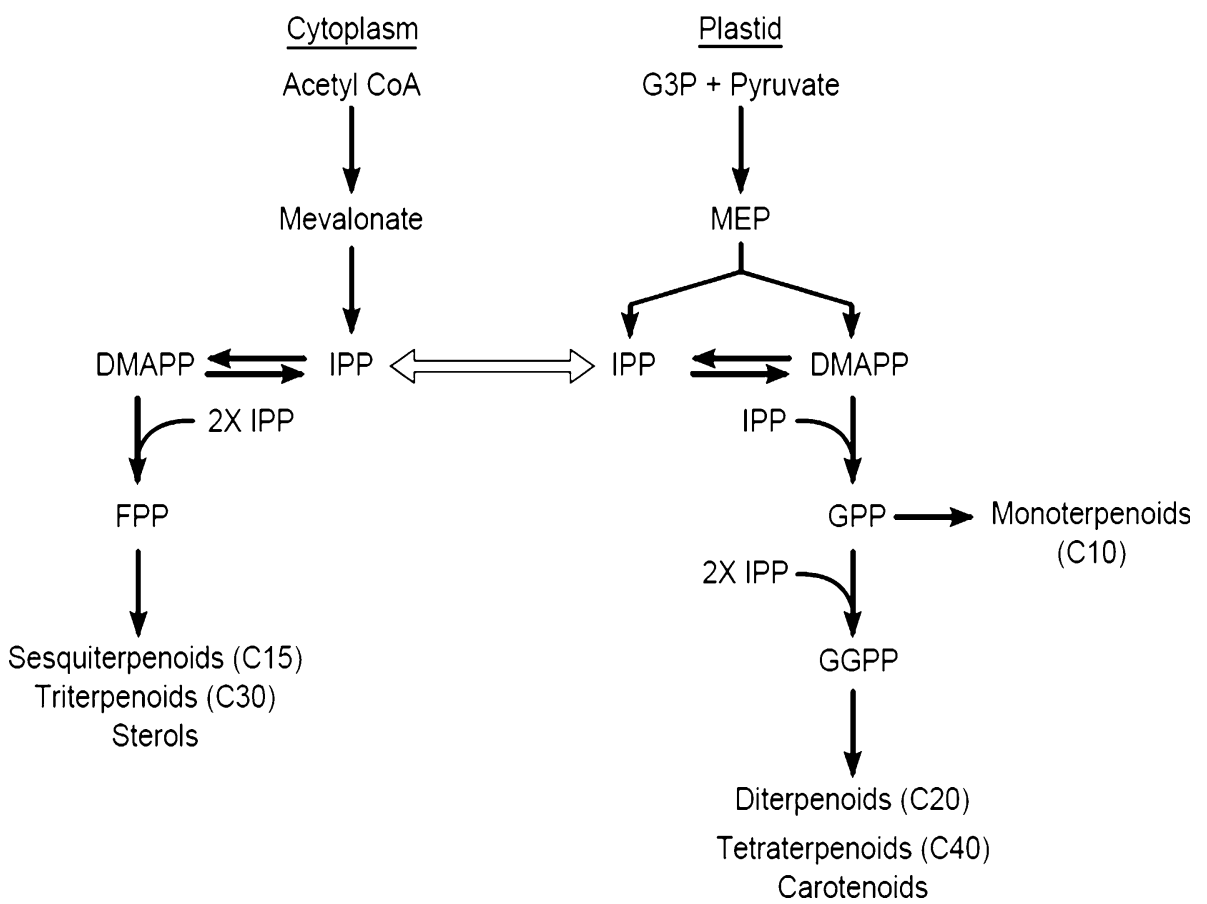

Plants require a long cultivation period and produce only low amounts of secondary metabolites. Therefore, cell culture systems are explored as an alternative source of valuable plant metabolites. Despite some successful examples (review in Verpoorte et al. 2002), the production of secondary metabolites in most plant cell cultures is relatively low or sometimes zero. In the case of $C$. roseus, bisindole alkaloids such as vincristine and vinblastine are not produced in cell cultures due to the lack of one of the biosynthetic precursor, i.e., vindoline. Nevertheless, semisynthesis of bisindole alkaloids is possible by coupling catharanthine and vindoline, thus optimizing cell or organ cultures to produce the individual precursors is an interesting target. Although ajmalicine and serpentine accumulate in $C$. roseus cell cultures, the productivity is too low compared to the intact plants for a cost-competitive mass production (Zhao and Verpoorte 2007).

Several strategies can be applied to improve product yields in plant cell cultures. One of the techniques is to induce the biosynthesis of secondary metabolites by elicitation. Elicitation strategies using exogenous jasmonic acid (JA) or its volatile methyl ester, i.e., methyl jasmonic (MeJA), often result in elevated levels of certain secondary metabolites. In the elicitation process, jasmonates play an important role as regulatory signals to induce de novo transcription and translation leading to the induction of secondary metabolite biosynthesis in plant cell cultures (Memelink et al. 2001; Zhao and Verpoorte 2007). Exogenous application of jasmonates to $C$. roseus cell cultures (El-Sayed and Verpoorte 2002; Lee-Parsons and
Royce 2006; Vázquez-Flota et al. 2009), hairy roots (Rijhwani and Shanks 1998; Vázquez-Flota et al. 2009), shoot cultures (Vázquez-Flota et al. 2009) and seedlings (ElSayed and Verpoorte 2004) increased the production of TIA caused by an elevated expression of a set of biosynthesis related genes (Memelink et al. 2001). Combination of transcript and metabolic profiling of jasmonate elicited C. roseus cell cultures yielded a collection of known and previously undescribed transcript tags and metabolites associated with TIA (Rischer et al. 2006). Therefore, JA treatment is a useful biochemical tool to stimulate the production and to study the regulation of TIA biosynthesis.

TIAs are formed by the coupling of the indole tryptamine and the iridoid secologanin. The iridoid precursor originating from geranyl diphosphate (GPP; C10) is considered as the limiting factor of TIA biosynthesis in $C$. roseus cell cultures (Moreno et al. 1993; Whitmer et al. 2002). GPP is synthesized from the five-carbon (C5) precursors isopentenyl diphosphate (IPP) and dimethylallyl diphosphate (DMAPP), in which further condensation of these precursors generate farnesyl diphosphate (FPP; C15) and geranylgeranyl diphosphate (GGPP; C20), leading towards the biosynthesis of phytosterols (C30) and carotenoids (C40), respectively (Fig. 1). Apparently, the iridoid-terpenoid moiety of TIA is linked with other terpenoid pathways and several studies showed an interaction between the precursor pools in the cytosolic mevalonate and plastidial methyl-erythritol phosphate (MEP) pathways (Schuhr et al. 2003; Hemmerlin et al. 2003; Laule et al. 2003). This suggests that TIA production might be limited 
by competition for the $\mathrm{C} 5$ precursors and raises the question whether the stimulation of TIA production by certain signal molecules is due to a redistribution of precursors between the associated terpenoid pathways and/or to a total increase of precursor availability.

In this study, the effect of JA elicitation on different terpenoid pathways, i.e., monoterpenoids (TIA; C10), triterpenoids (phytosterols; C30), and tetraterpenoids (carotenoids; C40), and the distribution of C5 precursors into representative terpenoid groups were evaluated. The transient accumulation of TIAs, carotenoids, and phytosterols were analyzed by high performance liquid chromatographydiode array detector (HPLC-DAD) or gas chromatographyflame ionization detector (GC-FID). Furthermore, the metabolomic changes between control and treated samples were analyzed by NMR to portray metabolic alteration in JA-elicited $C$. roseus cell cultures.

\section{Materials and methods}

\section{Cell culture materials}

The experiments were made with the Leiden University CRPP-glucose cell line of $C$. roseus (Natural Products Laboratory, Institute of Biology, Leiden University, The Netherlands). The cell suspension culture was subcultured every 3 weeks by diluting the cultures with fresh medium (1:1) consisting of B5 medium (Gamborg et al. 1968) supplemented with $1.86 \mathrm{mg} / \mathrm{l} \mathrm{NAA}$ and $20 \mathrm{~g} / \mathrm{l}$ glucose. The medium was adjusted to $\mathrm{pH} 5.8$ before autoclaving at $121{ }^{\circ} \mathrm{C}$ for $20 \mathrm{~min}$. The cultures were maintained in $250 \mathrm{ml}$ shake flasks closed with T-32 silicon foam stoppers (Shin Etsu, Tokyo, Japan) and placed on a gyratory shaker (110 rpm) at $25^{\circ} \mathrm{C}$ in continuous light $\left(10.8-27 \mu \mathrm{E} / \mathrm{m}^{2} / \mathrm{s}\right)$. For the experiments, $20 \mathrm{ml}$ (approx. $4 \mathrm{~g}$ fresh weight) of a 2 -week-old cell suspension culture was inoculated into $50 \mathrm{ml}$ culture medium and cultivated as indicated until further treatment and/or harvesting.

\section{Elicitation}

The stock solution of JA $(10 \mathrm{mg} / \mathrm{ml})$ was prepared by dissolving JA (Sigma-Aldrich Chemie, Steinheim, Germany) in $2 \mathrm{ml}$ ethanol and diluted with sterile water to acquire the total volume of $10 \mathrm{ml}$. The solution was mixed and filter sterilized through $0.22 \mu \mathrm{m}$ Millex $^{\mathrm{TM}}$ filter (Millipore, Bedford, MA, USA). At the fifth day of culture, $50 \mu \mathrm{l}$ of JA solution was added into the cell culture $(70 \mathrm{ml})$ to achieve the final concentration of $34 \mu \mathrm{M}(0.5 \mathrm{mg} \mathrm{JA} /$ flask). The same amount of the solvent $(20 \% \mathrm{v} / \mathrm{v}$ ethanol) was applied to the cell cultures as control treatment. The JA-treated and control cultures were grown in triplicate for every harvesting time points $(0,2,6,24,48$, and $72 \mathrm{~h}$ after elicitation). The cells were filtered under reduced pressure, washed three times with deionized water, and lyophilized for $72 \mathrm{~h}$.

\section{Analysis of terpenoid indole alkaloids, carotenoids, and sterols}

Analysis of TIAs, carotenoids, and sterols were carried out according to the methods described by Saiman et al. (2014). Briefly, $100 \mathrm{mg}$ of the freeze-dried cells were weighed and extracted for analyzing TIA and sterols, while $70 \mathrm{mg}$ freeze-dried cells were extracted for carotenoids. Analyses of carotenoids, TIA and TIA precursors were performed using different HPLC-DAD methods, whereas the GC-FID system was used to analyze sterols. Calibration curves of the standard compounds were made for quantitative analyses. Results were presented as an average of the analyses of two separate flasks for several timepoints $(0,6,24$, and $72 \mathrm{~h}$ after treatment).

\section{NMR measurement}

NMR analysis was conducted according to the protocol of Kim et al. (2010). The freeze-dried cells $(25 \mathrm{mg}$ ) from triplicate flasks of each treatments (JA and control) and time-points $(0,2,6,24,48,72 \mathrm{~h})$ were extracted with $1.2 \mathrm{ml}$ of $\mathrm{CD}_{3} \mathrm{OD}$ and $0.3 \mathrm{ml}$ of $\mathrm{KH}_{2} \mathrm{PO}_{4}$ buffer in $\mathrm{D}_{2} \mathrm{O}$ (pH 6.0, containing $0.01 \% \mathrm{w} / \mathrm{w}$ trimethylsilyl propanoic acid (TMSP) as internal standard). The mixture was vortexed for $10 \mathrm{~s}$, sonicated for $10 \mathrm{~min}$, and centrifuged for $15 \mathrm{~min}(14,000 \mathrm{rpm})$. The supernatant was transferred into an NMR tube for measurement of ${ }^{1} \mathrm{H}-\mathrm{NMR}$ using a Bruker AV $600 \mathrm{MHz}$ spectrometer (Bruker, Karlsruhe, Germany) with cryoprobe. The ${ }^{1} \mathrm{H}$-NMR spectra were recorded at $25^{\circ} \mathrm{C}$, consisted of 128 scans requiring $10 \mathrm{~min}$ and $26 \mathrm{~s}$ acquisition time with following parameters: $0.16 \mathrm{~Hz} /$ point, pulse width of $30(11.3 \mu \mathrm{s})$, and relaxation delay of $1.5 \mathrm{~s}$. $\mathrm{CD}_{3} \mathrm{OD}$ was used as the internal lock. A presaturation sequence was used to suppress the residual water signal with low power selective irradiation at the water frequency during the recycle delay. Free induction decay was Fourier transformed with a line-broadening (LB) factor of $0.3 \mathrm{~Hz}$.

The resulting spectra were manually phased, baseline corrected, and calibrated to TMSP at $0.0 \mathrm{ppm}$ by using XWIN NMR version 3.5 (Bruker). The AMIX software (Bruker) was used to reduce the ${ }^{1} \mathrm{H}-\mathrm{NMR}$ spectra to an ASCII file, with total intensity scaling. Bucketing or binning was performed and the spectral data were reduced to include regions of equal width $(\delta 0.04)$ corresponding to the region of $\delta 0.40-10.00$. The regions of $\delta 4.85-4.95$ and $\delta 3.25-3.35$ were not included in the analysis because of the remaining signal of $\mathrm{D}_{2} \mathrm{O}$ and $\mathrm{CD}_{3} \mathrm{OD}$, respectively. 

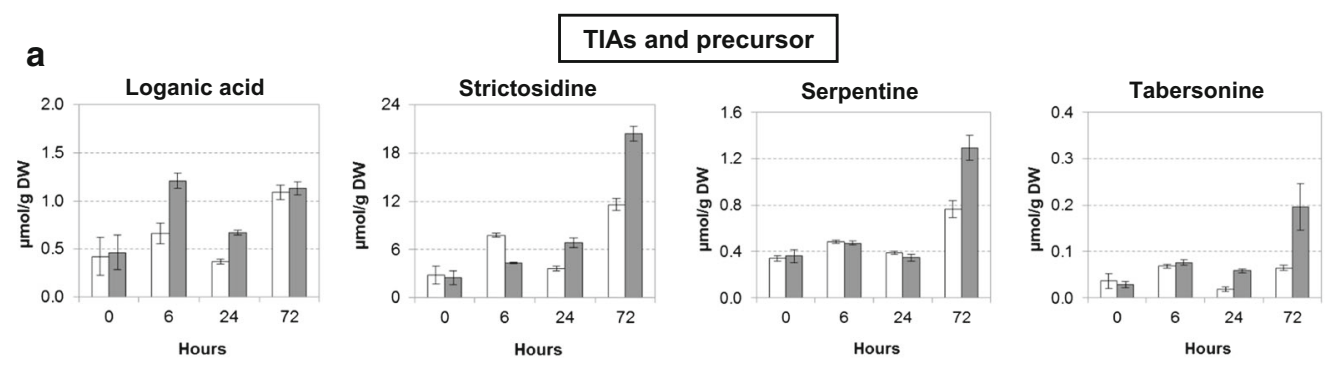

\section{$\square$ Control}

$\square$ Jasmonate
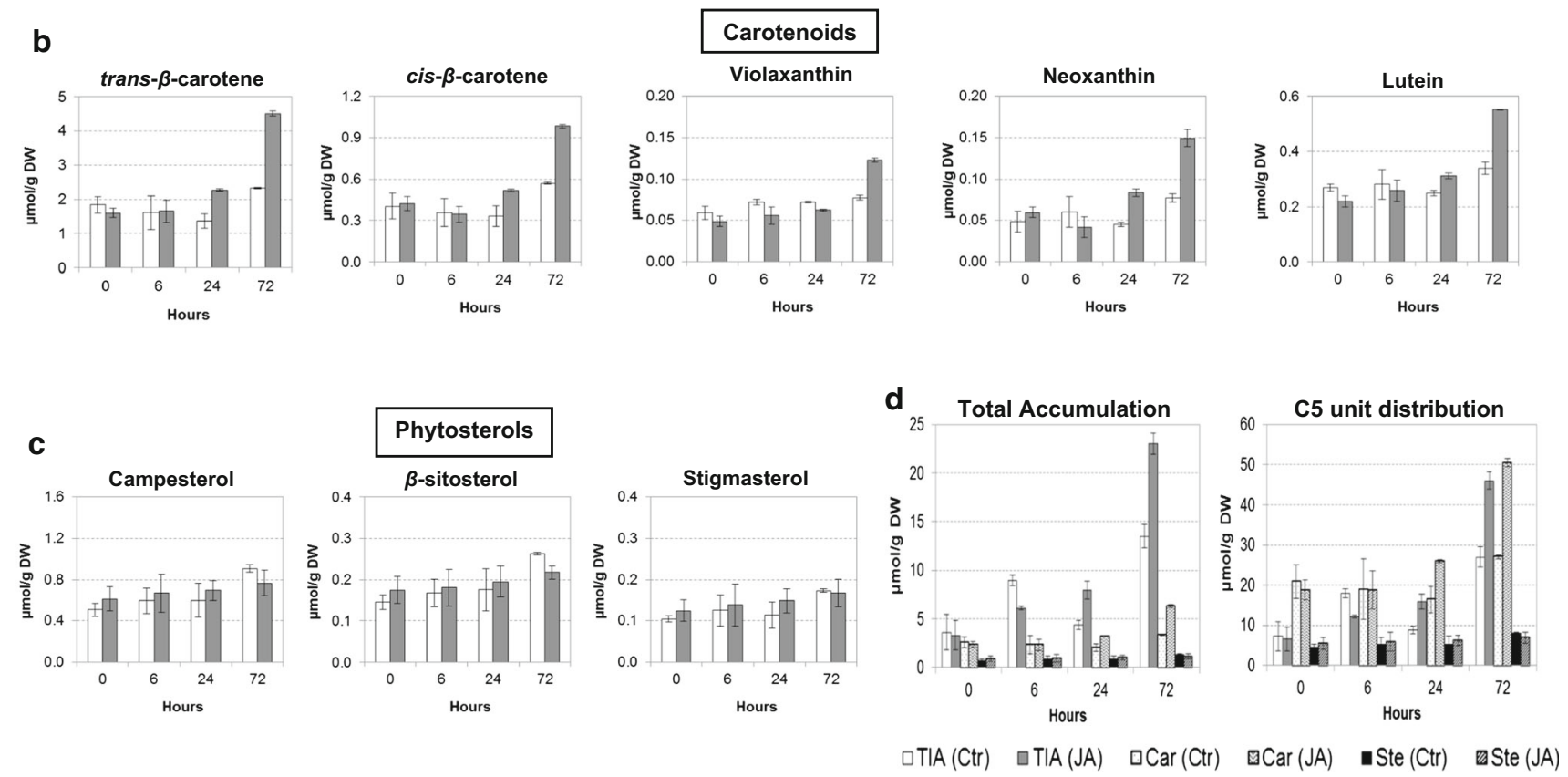

Fig. 2 Time-course of loganic acid and TIAs (a) carotenoids (b), and phytosterols (c) production measured by HPLC-DAD and GC-FID, respectively, in CRPP cell line elicited with $34 \mu \mathrm{M}$ JA or control

solution. Total accumulation and distribution of C5 precursors after elicitation (d). Results are the mean of two replicates; error bars indicate the two values

Multivariate data analysis was performed with the SIMCA$\mathrm{P}+$ software version 12.0 (Umetrics, Umeå, Sweden). The $t$ test was performed using IBM SPSS Statistics 20 (SPSS Inc., Chicago, IL, USA) to determine statistical significance $(P<0.05)$ of the relative levels of metabolites.

\section{Results and discussion}

\section{Cell culture material}

Nine lines of $C$. roseus cell suspension cultures were analyzed for the accumulation of different terpenoid groups, i.e., TIA, carotenoids, and sterols. Of the C. roseus cell lines, the CRPP cell lines were found to have high productivity in terms of quantity and diversity of TIA and terpenoid compounds (Saiman et al. 2014). Therefore, the CRPP cell line was chosen as a model; in particular the glucose supplemented CRPP cell line was used for this study for its suitability to feed with labeled glucose to study the carbon fluxes in the future.

Prior to the elicitation study, the growth curve of the CRPP (glucose) cell line was determined to estimate the best time-point for elicitation. Based on the cell growth curve (Supplementary 1), the elicitation experiment was performed at day 5 , when the maximum biomass is reached and high metabolic rates can be achieved.

\section{Analysis of terpenoid indole alkaloids and precursors}

The analysis of iridoids showed only the presence of loganic acid, whereas loganin and secologanin, if present, were below detection limit. Neither tryptophan nor tryptamine was detected in the CRPP cell line. TIA analysis only showed strictosidine as major alkaloid and smaller amounts of serpentine, and tabersonine. Figure 2a shows the levels of loganic acid and TIAs at different time-points 
after JA elicitation. Loganic acid was twofold higher in the JA-elicited cells $6 \mathrm{~h}$ after elicitation. Although loganic acid concentration was still higher compared to the control at $24 \mathrm{~h}$, the level was lower than at $6 \mathrm{~h}$ after JA elicitation. At the later time-points, loganic acid levels in both elicited and control cells were not different. The central intermediate of TIA, strictosidine, was about twofold increased at 24 and $72 \mathrm{~h}$ after elicitation, while the serpentine level was only increased at the latter time-point. The tabersonine level was also increased (threefold) at $72 \mathrm{~h}$ after elicitation. Overall, loganic acid concentration was increased between 6 and $24 \mathrm{~h}$ after elicitation and subsequently the levels of strictosidine and the other down-stream TIAs gradually increased in time.

The increased levels of TIA upon jasmonate elicitation result from elevated expression of all known genes encoding enzymes involved in TIA biosynthesis (van der Fits and Memelink 2000; Memelink et al. 2001). The jasmonate-responsive expression of a number of these biosynthesis genes is controlled by transcriptional regulators such as octadecanoid-derivative responsive Catharanthus AP2-domain (ORCA) proteins. ORCA3 gene expression was rapidly induced by jasmonates as early as $30 \mathrm{~min}$ after elicitation. Yet its expression level decreased after an hour and returned to the control level at $24 \mathrm{~h}$ after elicitation. The induced ORCA3 expression preceded induction of both tryptophan decarboxylase (TDC) and strictosidine synthase (STR) gene expression by at least $2 \mathrm{~h}$. In contrast to ORCA3, the expression levels of the latter continued to rise up to $8 \mathrm{~h}$ after elicitation. At $24 \mathrm{~h}$ after jasmonate elicitation, the expression levels of TDC and STR were decreased (van der Fits and Memelink 2001). In accordance to the TIA accumulation in this study, the kinetic pattern of TDC and STR transcript levels and metabolites show that the latter are delayed by at least $24 \mathrm{~h}$ after induction of gene transcription.

Besides the TDC and STR genes, C. roseus cell cultures constitutively overexpressing ORCA3 also showed induction of the expression of genes encoding the enzymes 1-deoxy-D-xylulose 5-phosphate synthase (DXS), anthranilate synthase (AS $\alpha$ ), cytochrome $\mathrm{P} 450$ reductase (CPR), and desacetoxyvindoline 4-hydroxylase (D4H), but not of geraniol 10-hydroxylase $(\mathrm{G} 10 \mathrm{H})$ (or also known as geraniol 8-oxidase; G8O), strictosidine $\beta$-D-glucosidase (SGD), and deacetylvindoline 4- $O$-acetyltransferase (DAT) (van der Fits and Memelink 2000). Apparently, not all jasmonate-induced genes are regulated by ORCA3. Another AP2/ERF-domain transcription factor called ORCA2 was found to induce the expression of STR (Menke et al. 1999). A study by Hasnain (2010) showed that inducible overexpression of the transcription factors ORCA2 or ORCA3 in $C$. roseus cell cultures induced the expression of the genes encoding AS $\alpha$, TDC, STR, D4H, loganic acid methyltransferase (LAMT) and secologanin synthase
(SLS) but not the genes encoding G10H and CPR, which might be controlled by different transcription factors. Memelink et al. (2001) suggested that ORCA2 and ORCA3 regulate different but overlapping sets of genes. Recently, all structural genes in the iridoid pathway of $C$. roseus have been discovered and all of the genes from geraniol synthase $(G E S)$ to $S L S$ are induced by jasmonate (Miettinen et al. 2014). However, it remains to be determined which transcription factor regulates these genes. In addition, information on the transient patterns of gene expressions in the iridoid pathway upon JA-elicitation are not yet available; a low expression of genes down-stream of loganic acid in the early time-points and induction of TIA at later time-points would explain the obtained loganic acid accumulation pattern and conversion to strictosidine. Additional expression data should be acquired to resolve the elements controlling the accumulation patterns of these and further down-stream metabolites.

It should be noted that the composition of strictosidine, serpentine, and tabersonine in the control CRPP cell line at the $72 \mathrm{~h}$ time-point was $93.3,6.1$, and $0.5 \%$ respectively, whereas after elicitation, those compounds were all increased but the relative composition was not really changed with strictosidine (93\%), serpentine $(6 \%)$, and tabersonine $(1 \%)$. This shows that the flux through the pathway is increased, but within the TIA biosynthetic network the enzyme levels and kinetic characteristics remain the same for the enzymes involved in the biosynthesis of serpentine and tabersonine, and apparently the activities are not fully used. This fits with the observations of Whitmer et al. (2002) who showed that by feeding TIA precursors the TIA network has a large overcapacity that under normal condition is not used.

\section{Analysis of carotenoids}

An increased level of carotenoids can be observed at $72 \mathrm{~h}$ after JA elicitation in the $C$. roseus cell suspension culture (Fig. 2b). At that time-point, the concentrations of all measured carotenoids in this study were about twofold higher in the JA-treated cells compared to the controls. However, the composition of the mixture of carotenoids in the $C$. roseus cell culture did not alter after JA treatment, i.e., $\beta$-carotene stands for the largest part of the total carotenoids followed by lutein, neoxanthin, and violaxanthin.

Previous studies showed that jasmonate regulates the biosynthesis of carotenoids (Pérez et al. 1993; Rudell et al. 2002; Liu et al. 2012). Jasmonate elicitation increased the expression level of a number of biosynthesis genes including DXS (van der Fits and Memelink 2000), encoding an enzyme of upstream part of the MEP pathway. Overexpressing the DXS gene in transgenic Escherichia coli resulted in an increased accumulation of the carotenoids 
lycopene (Matthews and Wurtzel 2000), $\beta$-carotene (Albrecht et al. 1999) or zeaxanthin (Albrecht et al. 1999; Matthews and Wurtzel 2000). Furthermore, the GGPPS transcript levels in Taxus canadensis cell suspension cultures (Hefner et al. 1998) and Corylus avellana leaves (Wang et al. 2010) were up-regulated after MeJA treatment, even though no significant change in GGPPS expression was observed in $C$. roseus cell suspension cultures (Thabet et al. 2012). Thabet et al. (2012) speculated that there are several homologues of GGPPS in C. roseus and only some of them may be induced by MeJA as exemplified in tomato (Lycopersicon esculentum), in which LeGGPPSI was induced by jasmonate acid but not the LeGGPPS2 (Ament et al. 2006).

Carotenoids are essential in photosynthesis to absorb light energy and protect chlorophylls from photo damage (review in Namitha and Negi 2010). Pérez et al. (1993) showed that MeJA stimulates chlorophyll degradation in 'Golden Delicious' apple peel and chlorophyll $a / b$ ratio decreases with increasing MJ exposure. In contrast, Rudell et al. (2002) found a significant increase of chlorophyll $b$, while the level of chlorophyll $a$ was relatively stable after MeJA treatment in 'Fuji' apples. Rudell et al. (2002) suggested that the different results are probably due to the light treatment which was not applied by Pérez et al. (1993). In agreement with Rudell et al. (2002), a notable increase of both chlorophylls $a$ and $b$ was observed at $72 \mathrm{~h}$ after JA elicitation in our $C$. roseus cell suspension cultures under light treatment (Supplementary 2). This maybe the result of elevated activity of light harvesting connected with the increased level of carotenoids at that time-point.

\section{Analysis of phytosterols}

The levels of campesterol, $\beta$-sitosterol, and stigmasterol were not relatively different between JA-elicited and control cells (Fig. 2c). This indicates that JA elicitation at the concentration applied did not alter the biosynthesis of phytosterols. Studies in Glycyrrhiza glabra cell cultures (Hayashi et al. 2003) and the adventitious root cultures of Panax ginseng (Lee et al. 2004) showed that MeJA treatment did not induce the transcription of the gene encoding cycloartenol synthase, the enzyme responsible for the first step in sterol biosynthesis. However, several studies showed contradictory results on phytosterols accumulation after MeJA elicitation in different plants or plant cell cultures. For example, phytosterols production was enhanced in Lemna paucicostata cultured plants (Suh et al. 2013) and Capsicum апnиum cell culture (Sabater-Jara et al. 2010), decreased in Centella asiatica and Ruscus aculeatus cultured plants (Mangas et al. 2006), and remained constant in Galphimia glauca cultured plants (Mangas et al. 2006) and G. glabra cell cultures (Hayashi et al. 2003). The different results may indicate the existence of different control mechanisms among the plant species.

\section{Total accumulation and $\mathrm{C5}$ distribution}

The effect of JA elicitation in terms of total accumulation of the various terpenoid groups and the distribution of $\mathrm{C} 5$ precursors (Fig. 2d) were evaluated according to Saiman et al. (2014). In terms of total accumulation of the terpenoid groups, monoterpenoids consisting of loganic acid and several TIAs were highly accumulated during the time-course and after elicitation compared to carotenoids and sterols. The major contribution to the total composition of monoterpenoid accumulation comes from strictosidine (82\%), which indicates that the flux towards strictosidine is highly active in this specific $C$. roseus cell line. Total phytosterol content was relatively constant, whereas total carotenoids level was increased at 24 and $72 \mathrm{~h}$ after JA elicitation. These results suggest a relationship between TIA and carotenoid pathways induction as can be explained by up-regulation of several genes encoding enzymes in the MEP, TIA, and carotenoid pathways upon jasmonate elicitation (Thabet et al. 2012; van der Fits and Memelink 2000).

As regards to the distribution of C5 precursors, the level of C5 units increased in both carotenoids and TIA after 24 and $72 \mathrm{~h}$ of elicitation, whereas phytosterols did not change compared to the control. This shows that upon jasmonate elicitation, the carbon flow is activated towards the MEP pathway rather than the MVA pathway leading to phytosterols, and that export of IPP::DMAPP intermediates to the cytosolic terpenoid routes like the phytosterol is minimal. At $24 \mathrm{~h}$ after elicitation, the level of the C5 unit in TIAs was 1.8 -fold higher in elicited cells $(15.86 \mu \mathrm{mol} / \mathrm{g} \mathrm{DW})$ compared to the control $(8.80 \mu \mathrm{mol} / \mathrm{g} \mathrm{DW})$, whereas in carotenoids a 1.6-fold increase of C5 units was observed in the elicited cells $(25.97 \mu \mathrm{mol} / \mathrm{g} \mathrm{DW})$ compared to the control $(16.45 \mu \mathrm{mol} / \mathrm{g}$ DW). Subsequently, after $72 \mathrm{~h}$ of elicitation, the distribution of the $\mathrm{C} 5$ units to these pathways was almost doubled. Interestingly, between and within these pathways the ratios of the compounds more or less remain the same as in the control. This points towards regulation at the very beginning of the MEP pathway producing more $\mathrm{C} 5$ units, which are then, by the existing biochemical machinery, converted to the various products resulting in similar ratios of these as in control cells. The results suggest that both carotenoid and TIA pathways benefit from an increased carbon flow in the MEP pathway, but that elicitation does not really change the carbon flux distribution between the two pathways. TIA production might thus be further increased by changing the $\mathrm{C} 5$ distribution into these pathways, e.g., by chemical inhibition of the carotenoid pathway and/or through metabolic engineering to reduce production of carotenoids. 


\section{Metabolic profiling by NMR}

The extracts of $C$. roseus cell suspension cultures harvested at different time-points after JA treatment and controls were subjected to ${ }^{1} \mathrm{H}-\mathrm{NMR}$ analysis. Figure 3 shows the comparison of ${ }^{1} \mathrm{H}-\mathrm{NMR}$ spectra of JA-elicited and control samples after $72 \mathrm{~h}$ treatment. The amino acids isoleucine, leucine, valine, and alanine were identified. Organic acids like acetic acid, succinic acid, malic acid, fumaric acid, and formic acid were also found. In the carbohydrate region, signals of anomeric protons of $\beta$-glucose, $\alpha$-glucose, and sucrose were detected. The aromatic part of the ${ }^{1} \mathrm{H}-\mathrm{NMR}$ spectra showed signals of indole moiety (twin triplets and doublets around $\delta 7.00-\delta 7.50)$ and the presence of signals at $\delta 7.07(t, J=7.8 \mathrm{~Hz}, \mathrm{H}-10), \delta 7.16(t, J=7.8 \mathrm{~Hz}$, $\mathrm{H}-11), \delta 7.37(d, J=8.0 \mathrm{~Hz}, \mathrm{H}-12), \delta 7.49(d, J=8.0 \mathrm{~Hz}$, $\mathrm{H}-9), \delta 7.80(s, \mathrm{H}-17)$, and $\delta 5.80\left(d, J=8.0 \mathrm{~Hz}, \mathrm{H}-1^{\prime}\right)$ represent characteristic signals of strictosidine. The other compound detected is loganic acid by the presence of signals at $\delta 1.08(d, J=6.0 \mathrm{~Hz}, \mathrm{H}-10), \delta 4.68(d$, $J=8.0 \mathrm{~Hz}, \mathrm{H}-7), \delta 5.27(d, J=4.0 \mathrm{~Hz}, \mathrm{H}-1), \delta 7.08(s$, $\mathrm{H}-3)$. The assignments of ${ }^{1} \mathrm{H}-\mathrm{NMR}$ signals were performed by comparing the reference spectra of our in-house database and the ${ }^{1} \mathrm{H}-\mathrm{NMR}$ signals reported by Kim et al. 2006 and Mustafa et al. 2009.

\section{Multivariate data analysis of NMR data}

Chemometric methods were applied to analyze the changes in the metabolic profiles between JA-elicited and control cells of $C$. roseus. Principal component analysis (PCA) is the most common method used to analyze data that arises from more than one variable. In this study, PCA was applied to the bucketed data of ${ }^{1} \mathrm{H}-\mathrm{NMR}$ spectra. The chemical shifts (ppm) were used as variables and the samples served as observations in the data matrix. The data was mean-centered and variables were scaled to the Pareto method.

The PCA score plot of the samples shows that the samples were separated due to the growth cycle; the older samples moved to the negative part of PC1 (Supplementary 3a). This indicates that the metabolite profiles change with the developmental status of the cells. It did not exhibit a clear distinction at $0-6 \mathrm{~h}$ after elicitation but a separation of the elicited and control samples was observed beyond $24 \mathrm{~h}$. As PCA is an unbiased method that shows maximum variation within the samples, a clear separation is not observed if the biological variation among the replicates is larger than between the groups. This was in fact found here for the early time-point samples and as experienced by Ali et al. (2012) in infected and control samples of grapevine at
Fig. $3{ }^{1} \mathrm{H}-\mathrm{NMR}$ spectra of jasmonate-elicited (red) and control (blue) cell suspension cultures of $C$. roseus at 72 h. 1 isoleucine, 2 leucine, 3 valine, 4 alanine, 5 acetic acid, 6 succinic acid, 7 malic acid, 8 sucrose (fructose moiety: $\delta 4.14$, glucose moiety: $\delta$ 5.41), 9 glucose ( $\beta$-glucose: $\delta 4.53, \alpha$ glucose: $\delta$ 5.15), 10 strictosidine, 11 loganic acid, 12 fumaric acid, 13 formic acid. (Color figure online)
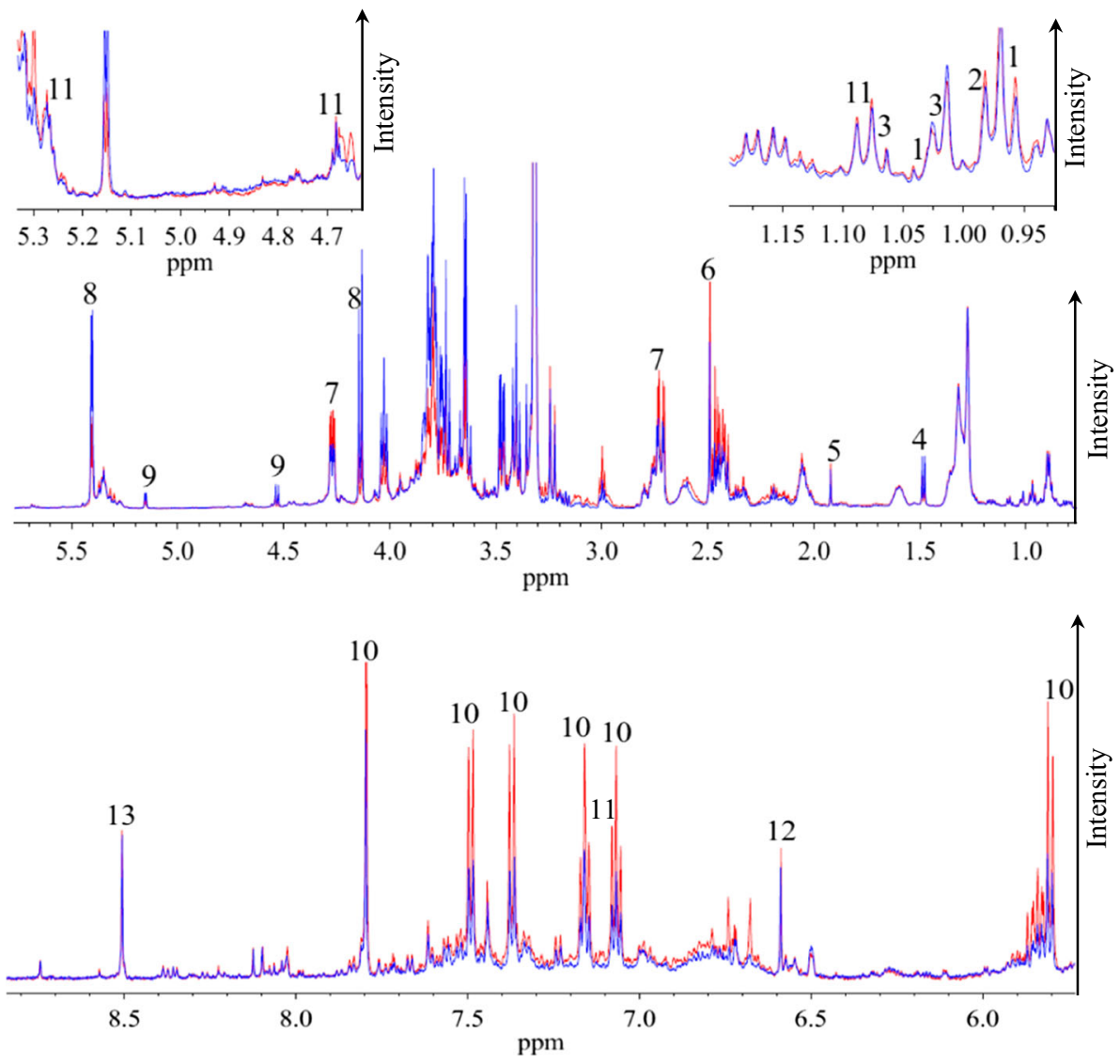

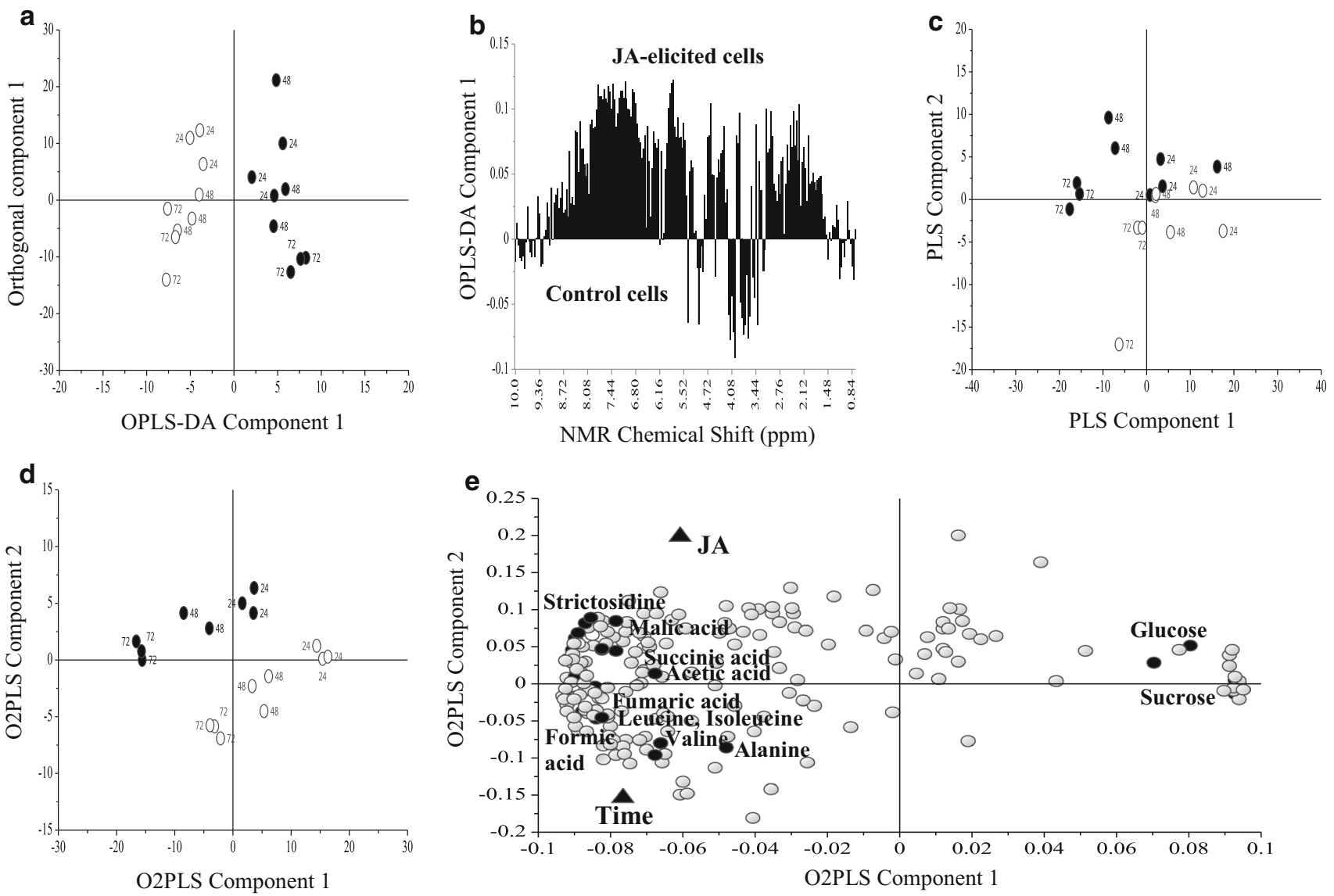

Fig. 4 Multivariate data analysis ( $\left.{ }^{1} \mathrm{H}-\mathrm{NMR}\right)$ of JA-elicited (filled circle) and control (open circle) samples of the CRPP cell line. Orthogonal projection to latent structures-discriminant analysis (OPLS-DA) score plot (a), OPLS-DA loadings plot (b), partial least

different time intervals. An effect of the developmental stage on the metabolome was also found in our C. roseus cell-line A12A2 when studying the time-effect associated to salicylic acid elicitation (Mustafa et al. 2009).

To extract information about specific variables, a supervised multivariate analysis is needed. Thus, partial least squares-discriminant analysis (PLS-DA) was applied to the same bucketed ${ }^{1} \mathrm{H}-\mathrm{NMR}$ spectra. In addition to the $\mathrm{X}$-matrix of NMR data, two classes (JA-treated and control cells) were assigned for the Y-matrix in PLS-DA. The variables were mean-centered and scaled to unit variance. However, PLS-DA was unable to give any significant model that fitted to the data. It was decided to separate the samples into two groups; 0-6 and 24-72 $\mathrm{h}$ since the earlier result in PCA shows such separation between JA elicitation and control samples beyond $24 \mathrm{~h}$ of elicitation. As expected, the PLS-DA of the samples at 0-6 h time-points did not deliver any fit component model, which indicates no significant separation among the samples. Instead, the samples at 24-72 $\mathrm{h}$ time-points show a separation between squares (PLS) score plot (c), bidirectional orthogonal projection to latent structures (O2PLS) score plot (d), and O2PLS loading plot (e). The numbers in the score plot are harvesting time (h) after treatments

JA-treated and control samples (Supplementary 3b). This model was validated by the permutation method through 100 applications, in which the Q2 values of permuted Y vectors were lower than the original ones and the regression of Q2 lines intersect at below zero. There is one outlier observed for the JA-treated samples at $48 \mathrm{~h}$ after elicitation, which could be due to either technical or biological variation of the sample; the latter is the most influential in many cases.

To get a better separation of the validated PLS-DA model, orthogonal projection to latent structures-discriminant analysis (OPLS-DA) was applied to the same data. OPLS-DA is used as improvement of the PLS-DA method to separate two or more classes of multivariate data (Bylesjö et al. 2006). As shown in Fig. 4a, OPLS-DA clearly distinguished between JA-treated and control samples after 24-72 h of elicitation. The loadings plot of OPLS-DA (Fig. 4b) reveals that the JA-elicited cells were higher in aromatic and amino/organic acid regions, while the control cells were distinguished by high levels of 

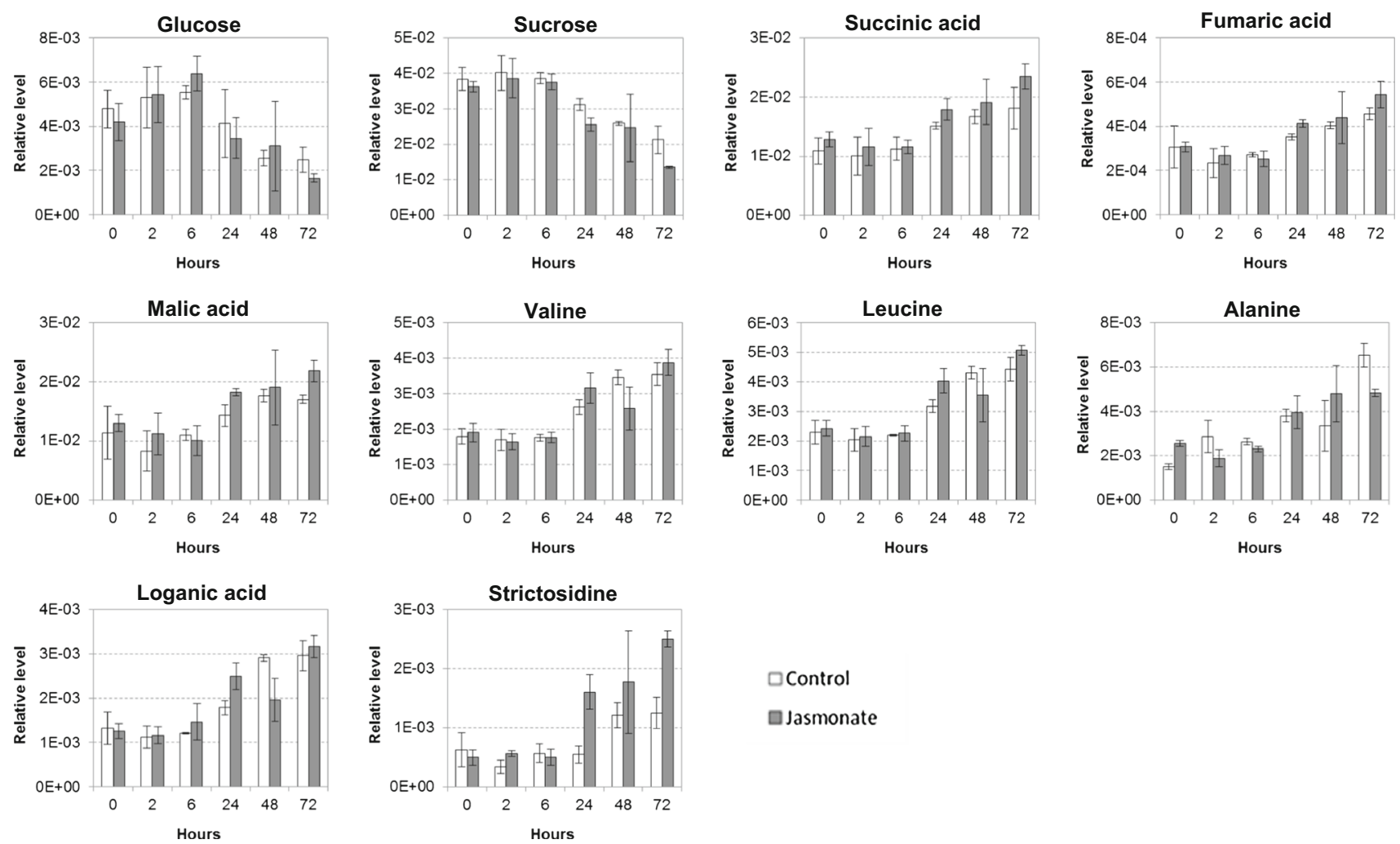

Fig. 5 Relative level of metabolites based on the mean area $\left({ }^{1} \mathrm{H}-\mathrm{NMR}\right.$ bucket data) of the resonance peak related to the metabolites. Results are the mean of three replicates; error bars indicate standard deviation

sugars. Some of the compounds identified for the separation of JA-elicited cells were strictosidine, succinic acid, fumaric acid, malic acid, and leucine. Sucrose, glucose, and alanine were found higher in the control samples. The increased level of strictosidine and decreased level of sugars in JA-elicited cells suggest that sugars are used for the production of de-novo biosynthesis of defense related compounds and the ATP needed in these biosynthetic pathways.

Another supervised algorithm, partial least square (PLS) can be used to reduce the dimensionality of multivariate data and to find relations between X matrices (metabolites) and $\mathrm{Y}$ matrices (time and treatment). As for the PLS-DA, only the 24-72 $\mathrm{h}$ samples deliver a good PLS model (Fig. 4c). Bidirectional orthogonal PLS (O2PLS) was further used to separate the treated and control samples. However, the sample separation in O2PLS showed a similar result as in the PLS model. By removing the fore mentioned outlier of the $48 \mathrm{~h}$ JA-elicited samples, the O2PLS model was improved (Fig. 4d). The O2PLS loading plot (Fig. 4e) shows that most of the metabolites were relatively increased through time except sugars. Strictosidine, malic acid, and succinic acid were most influenced by JA elicitation over the time period.

\section{Relative levels of metabolites detected by NMR}

The time-course levels of some metabolites identified by ${ }^{1} \mathrm{H}-$ NMR signals are shown in Fig. 5. As indicated by OPLS-DA and O2PLS results, sugar levels decreased by time; sucrose levels were significantly lower at 24 and $72 \mathrm{~h}$ in the JAelicited cells compared to the control $(P<0.05)$. In contrast, Flores-Sanchez et al. (2009) found no significant difference of sucrose and glucose levels in methyl jasmonate-elicited Cannabis sativa cell suspension cultures if compared to their respective controls; on the other hand, the elicitation treatment resulted in increased levels of fumaric acid in the $C$. sativa cells. In the $C$. roseus cells, some components of the tricarboxylic acid (TCA) cycle; succinic acid, fumaric acid, and malic acid were increased at 24 and $72 \mathrm{~h}$ after elicitation, though only malic acid levels were statistically significant compared to the control $(P<0.05)$. The current results suggest that upon jasmonate elicitation, sugars were catabolized and TCA cycle was activated to make building blocks and generate chemical energy for various biosynthetic routes that are part of the defense response.

The levels of valine remained statistically unaltered between JA-elicited and control cells at all time-points $(P<0.05)$. Leucine was found higher at 24 and $72 \mathrm{~h}$ after 
Fig. 6 Schematic summary of the results obtained in this study. The levels of the identified metabolites which were increased (blue), decreased (red), or relatively unchanged (grey) after JA elicitation in $C$. roseus cell suspension cultures. *Loganic acid level increased at $24 \mathrm{~h}$ after elicitation, whereas at $72 \mathrm{~h}$ the level is about similar to the level of the control. (Color figure online)

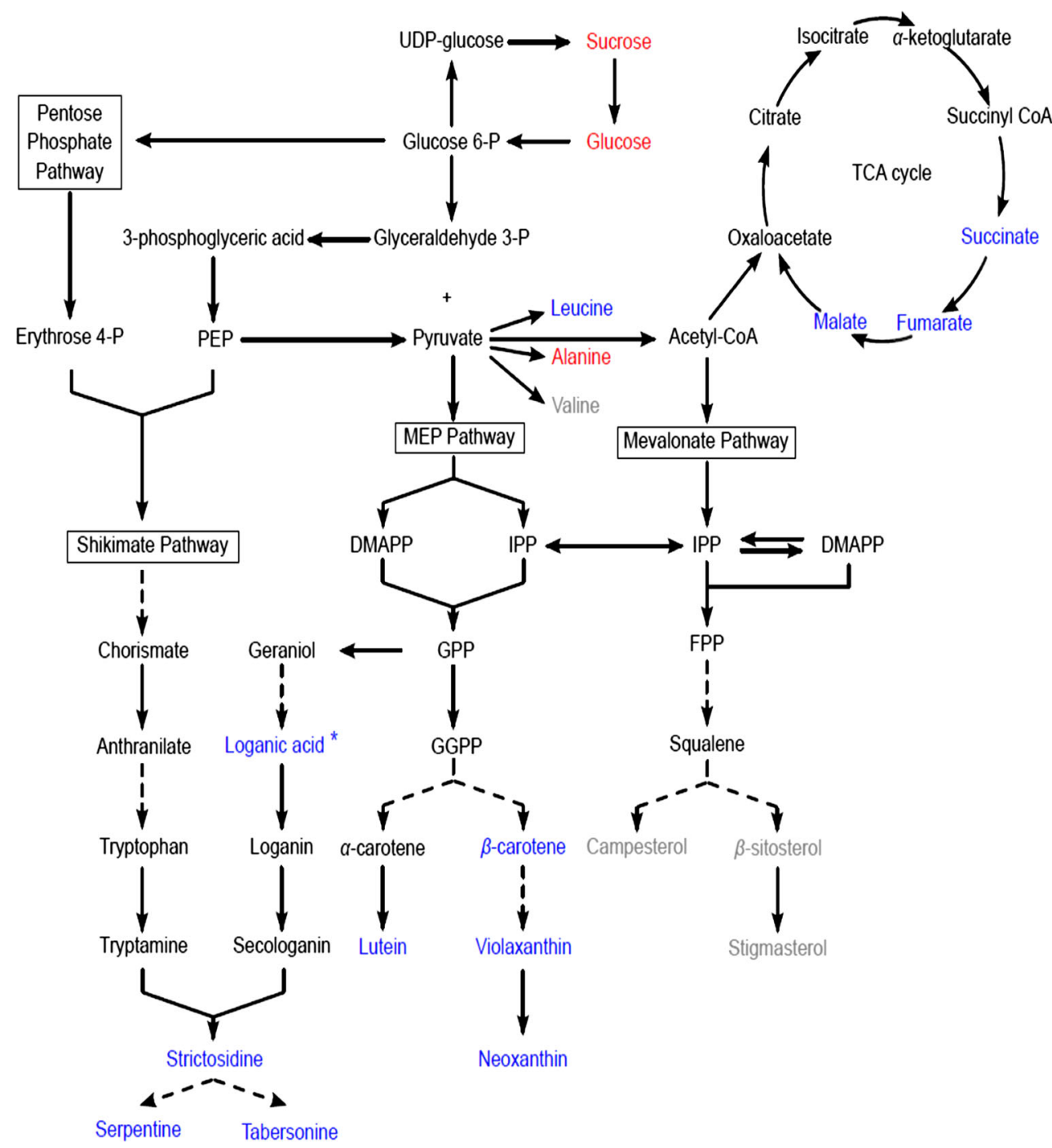

elicitation, in which the level at the former time-point was significantly increased $(P<0.05)$. Alanine was significantly decreased after $72 \mathrm{~h}$ of elicitation $(P<0.05)$, and this might be linked to the decreased levels of sugars. Similarly, Liang et al. (2006) found that glucose, sucrose, and alanine were decreased in MeJA treated Brassica rapa leaves compared to the control. Strictosidine levels were significantly increased at 24 and $72 \mathrm{~h}$ after elicitation $(P<0.05)$. The level of loganic acid in JA-elicited cells increased at $24 \mathrm{~h}(P<0.05)$, but the level was about the same as in the control cells at $72 \mathrm{~h}$. The results of strictosidine and loganic acid analyzed by NMR are in agreement to the results analyzed by HPLC.

\section{Conclusion}

This study is the first that describes the channeling of C5 units in the different terpenoid pathways in C. roseus cell suspension cultures upon JA elicitation. In the elicited cells, the levels of TIA and carotenoids were increased, while sterol accumulation was not affected, indicating that the flux of C5 units was only increased in the MEP pathway. It is suggested that the increased production of TIA and carotenoid is preferably due to the increase of the precursor availability rather than redistribution of $\mathrm{C} 5$ units between the associated terpenoid pathways. For both carotenoid and TIA pathways, the available enzymatic machinery seems to have an overcapacity, able to deal with increased carbon flux from the MEP pathway. To improve TIA production, channeling the increased availability of the C5 units away from carotenoids to TIA would be an interesting strategy.

NMR-based metabolomics is a powerful tool to simultaneously analyze both primary and secondary metabolites. In combination with multivariate data analysis, this approach is able to portray metabolic alteration in JA $C$. roseus cell cultures as summarized in Fig. 6. It reveals that the sugar levels were decreased, while the TCA cycle was 
activated to generate chemical energy and building blocks for a defense response, resulting in a higher accumulation of e.g., strictosidine, the precursor of many TIAs that may play a role in the plant defense system. Overall, this work shows that a systemic study of the metabolic changes in cells can lead to novel approaches of metabolic engineering.

Acknowledgments The authors thank the Ministry of Education Malaysia and University of Malaya, Kuala Lumpur, Malaysia for the financial support of Mohd Zuwairi Saiman. This research was funded by the IBOS-ACTS program as coordinated by NWO.

Open Access This article is distributed under the terms of the Creative Commons Attribution 4.0 International License (http:// creativecommons.org/licenses/by/4.0/), which permits unrestricted use, distribution, and reproduction in any medium, provided you give appropriate credit to the original author(s) and the source, provide a link to the Creative Commons license, and indicate if changes were made.

\section{References}

Albrecht M, Misawa N, Sandmann G (1999) Metabolic engineering of the terpenoid biosynthetic pathway of Escherichia coli for production of the carotenoids $\beta$-carotene and zeaxanthin. Biotechnol Lett 21:791-795

Ali K, Maltese F, Figueiredo A, Rex M, Forte AM, Zyprian E, Pais MS, Verpoorte R, Choi YH (2012) Alterations in grapevine leaf metabolism upon inoculation with Plasmopara viticola in different time-points. Plant Sci 191-192:100-107

Ament K, Van Schie CC, Bouwmeester HJ, Haring MA, Schuurink RC (2006) Induction of a leaf specific geranylgeranyl pyrophosphate synthase and emission of (E,E)-4,8,12-trimethyltrideca1,3,7,11-tetraene in tomato are dependent on both jasmonic acid and salicylic acid signaling pathways. Planta 224:1197-1208

Bylesjö M, Rantalainen M, Cloarec O, Nicholson JK, Holmes E, Trygg J (2006) OPLS discriminant analysis: combining the strengths of PLS-DA and SIMCA classification. J Chemometrics 20:341-351

El-Sayed MA, Verpoorte R (2002) Effect of phytohormones on growth and alkaloid accumulation by a Catharanthus roseus cell suspension culture fed with the alkaloid precursors tryptamine and loganin. Plant Cell Tissue Organ Cult 68:265-270

El-Sayed MA, Verpoorte R (2004) Growth, metabolic profiling and enzymes activities of Catharanthus roseus seedlings treated with plant growth regulators. Plant Growth Reg 4:53-58

Flores-Sanchez IJ, Peč J, Fei J, Young Hae Choi YH, Dušek J, Verpoorte R (2009) Elicitation studies in cell suspension cultures of Cannabis sativa L. J Biotechnol 143:157-168

Gamborg OL, Miller RA, Ojima K (1968) Nutrient requirements of suspension cultures of soybean root cells. Exp Cell Res 50:155-158

Hasnain G (2010) The ORCA-ome as a key to understanding alkaloid biosynthesis in Catharanthus roseus. $\mathrm{PhD}$ Thesis, Leiden University, The Netherlands. Chapter 2, Genome-wide determination of ORCA target genes in Catharanthus alkaloids, pp. 28-48. ISBN 9789085705826

Hayashi H, Huang P, Inoue K (2003) Up-regulation of soya saponin biosynthesis by methyl jasmonate in cultured cells of Glycyrrhiza glabra. Plant Cell Physiol 44:404-411
Hefner J, Ketchum RE, Croteau R (1998) Cloning and functional expression of a cDNA encoding geranylgeranyl diphosphate synthase from Taxus canadensis and assessment of the role of this prenyltransferase in cells induced for taxol production. Arch Biochem Biophys 360:62-74

Hemmerlin A, Hoeffler JH, Meyer O, Tritsch D, Kagan IA, Grosdemange-Billiard C, Rohmer M, Bach TJ (2003) Cross-talk between the cytosolic mevalonate and the plastidial methylerythritol phosphate pathways in Tobacco Bright Yellow-2 cells. J Biol Chem 18:26666-26676

Kim HK, Choi YH, Verpoorte R (2006) Metabolomic analysis of Catharanthus roseus using NMR and principal component analysis. In: Saito K, Dixon RA, Willmitzer L (eds) Biotechnology in agriculture and forestry. Plant metabolomics, vol 57. Springer, Leipzig, pp 261-276

Kim HK, Choi YH, Verpoorte R (2010) NMR-based metabolomic analysis of plants. Nat Protoc 5:536-549

Laule O, Fürholz A, Chang H-S, Zhu T, Wang X, Heifetz PB, Gruissem W, Lange BM (2003) Crosstalk between cytosolic and plastidial pathways of isoprenoid biosynthesis in Arabidopsis thaliana. Proc Natl Acad Sci USA 100:6866-6871

Lee MH, Jeong JH, Seo JW, Shin CG, Kim YS, In JG, Yang DC, Yi JS, Choi YE (2004) Enhanced triterpene and phytosterol biosynthesis in Panax ginseng overexpressing squalene synthase gene. Plant Cell Physiol 45:976-984

Lee-Parsons CWT, Royce AJ (2006) Precursor limitations in methyl jasmonate-induced Catharanthus roseus cell cultures. Plant Cell Rep 25:607-612

Liang YS, Choi YH, Kim HK, Linthorst HJM, Verpoorte R (2006) Metabolomic analysis of methyl jasmonate treated Brassica rapa leaves by 2-dimensional NMR spectroscopy. Phytochemistry 67:2503-2511

Liu L, Wei J, Zhang M, Zhang L, Li C, Wang Q (2012) Ethylene independent induction of lycopene biosynthesis in tomato fruits by jasmonates. J Exp Bot 63:5751-5761

Mangas S, Bonfill M, Osuna L, Moyano E, Tortoriello J, Cusido RM, Pinol MT, Javier Palazon J (2006) The effect of methyl jasmonate on triterpene and sterol metabolisms of Centella asiatica, Ruscus aculeatus and Galphimia glauca cultured plants. Phytochemistry 67:2041-2049

Matthews PD, Wurtzel ET (2000) Metabolic engineering of carotenoid accumulation in Escherichia coli by modulation of the isoprenoid precursor pool with expression of deoxyxylulose phosphate synthase. Appl Microbiol Biotechnol 53:396-400

Memelink J, Kijne JW, van der Heijden R, Verpoorte R (2001) Genetic modification of plant secondary metabolite pathways using transcriptional regulators. Adv Biochem Eng Biotechnol 72:103-125

Menke FL, Champion A, Kijne JW, Memelink J (1999) A novel jasmonate- and elicitor-responsive element in the periwinkle secondary metabolite biosynthetic gene Str interacts with a jasmonate- and elicitor-inducible AP2-domain transcription factor, ORCA2. EMBO J 18:4455-4463

Miettinen K, Dong L, Navrot N, Schneider T, Burlat V, Pollier J, Woittiez L, van der Krol S, Lugan R, Ilc T, Verpoorte R, Oksman-Caldentey KM, Martinoia E, Bouwmeester H, Goossens A, Memelink J, Werck-Reichhart D (2014) The seco-iridoid pathway from Catharanthus roseus. Nat Commun 5:3606

Moreno PRH, van der Heijden R, Verpoorte R (1993) Effect of terpenoid precursor feeding and elicitation on formation of indole alkaloids in cell suspension cultures of Catharanthus roseus. Plant Cell Rep 12:702-705

Mustafa NR, Kyong Kim Hye, Choi YH, Verpoorte R (2009) Metabolic changes of salicylic acid-elicited Catharanthus roseus cell suspension cultures monitored by NMR-based metabolomics. Biotechnol Lett 31:1967-1974 
Namitha KK, Negi PS (2010) Chemistry and biotechnology of carotenoids. Crit Rev Food Sci Nutr 50:728-760

Pérez AG, Sanz C, Richardson DG, Olías JM (1993) Methyl jasmonate vapor promotes $\beta$-carotene synthesis and chlorophyll degradation in Golden Delicious apple peel. J Plant Growth Regul 12:163-167

Rijhwani SK, Shanks JV (1998) Effects of elicitor dosage and exposure time on biosynthesis of indole alkaloids by Catharanthus roseus hairy root cultures. Biotechnol Prog 14:442-449

Rischer H, Oresic M, Seppänen-Laakso T, Katajamaa M, Lammertyn F, Ardiles-Diaz W, Van Montagu MCE, Inzé D, OksmanCaldentey KM, Goossens A (2006) Gene-to-metabolite networks for terpenoid indole alkaloid biosynthesis in Catharanthus roseus cells. Proc Natl Acad Sci USA 103:5614-5619

Rudell DR, Mattheis JP, Fan X, Fellman JK (2002) Methyl jasmonate enhances anthocyanin accumulation and modifies production of phenolics and pigments in 'Fuji' apples. J Am Soc Hortic Sci 127:435-441

Sabater-Jara AB, Almagroa L, Belchí-Navarroa S, Ferrerb MA, Barcelóa AR, Pedrenoa MA (2010) Induction of sesquiterpenes, phytoesterols and extracellular pathogenesis-related proteins in elicited cell cultures of Capsicum annuиm. J Plant Physiol 167:1273-1281

Saiman MZ, Mustafa NR, Pomahocova B, Verberne M, Verpoorte R, Choi YH, Schulte AE (2014) Analysis of metabolites in the terpenoid pathway of Catharanthus roseus cell suspensions. Plant Cell Tissue Organ Cult 117:225-239

Schuhr CA, Radykewicz T, Sagner S, Latzel C, Zenk MH, Arigoni D, Bacher A, Rohdich F, Eisenreich W (2003) Quantitative assessment of crosstalk between the two isoprenoid biosynthesis pathways in plants by NMR spectroscopy. Phytochem Rev $2: 3-16$

Suh WH, Hyun SH, Kim SH, Lee SY, Choi HK (2013) Metabolic profiling and enhanced production of phytosterols by elicitation with methyl jasmonate and silver nitrate in whole plant cultures of Lemna paucicostata. Process Biochem 48:1581-1586
Thabet I, Guirimand G, Guihur A, Lanoue A, Courdavault V, Papon N, Bouzid S, Giglioli-Guivarc'h N, Simkin AJ, Clastre M (2012) Characterization and subcellular localization of geranylgeranyl diphosphate synthase from Catharanthus roseus. Mol Biol Rep 39:3235-3243

van der Fits L, Memelink J (2000) ORCA3, a jasmonate-responsive transcriptional regulator of plant primary and secondary metabolism. Science 289:295-297

van der Fits L, Memelink J (2001) The jasmonate-inducible AP2/ ERF-domain transcription factor ORCA3 activates gene expression via interaction with a jasmonate-responsive promoter element. Plant J 25:43-53

van der Heijden R, Jacobs DI, Snoeijer W, Hallard D, Verpoorte R (2004) The Catharanthus alkaloids: pharmacognosy and biotechnology. Curr Med Chem 11:607-628

Vázquez-Flota F, Hernández-Domínguez E, de Miranda-Ham ML, Monforte-González M (2009) A differential response to chemical elicitors in Catharanthus roseus in vitro cultures. Biotechnol Lett 31:591-595

Verpoorte R, Contin A, Memelink J (2002) Biotechnology for the production of plant secondary metabolites. Phytochem Rev 1:13-25

Wang Y, Miao Z, Tang K (2010) Molecular cloning and functional expression analysis of a new gene encoding geranylgeranyl diphosphate synthase from hazel (Corylus avellana L. Gasaway). Mol Biol Rep 37:3439-3444

Whitmer S, van der Heijden R, Verpoorte R (2002) Effect of precursor feeding on alkaloid accumulation by a strictosidine synthase over-expressing transgenic cell line $\mathrm{S} 1$ of Catharanthus roseus. Plant Cell Tissue Organ Cult 69:85-93

Zhao J, Verpoorte R (2007) Manipulating indole alkaloid production by Catharanthus roseus cell cultures in bioreactors: from biochemical processing to metabolic engineering. Phytochem Rev 6:435-457 